\title{
Eribulin versus dacarbazine in previously treated patients with advanced liposarcoma or leiomyosarcoma: a randomised, open-label, multicentre, phase 3 trial
}

\begin{abstract}
Patrick Schöffski, Sant Chawla, Robert G Maki, Antoine Italiano, Hans Gelderblom, Edwin Choy, Giovanni Grignani, Veridiana Camargo, Sebastian Baver, Sun Young Rha, Jean-Yves Blay, Peter Hohenberger, David D’Adamo, Matthew Guo, Bartosz Chmielowski, Axel Le Cesne, George D Demetri, Shreyaskumar R Patel
\end{abstract}

\begin{abstract}
Summary
Background A non-randomised, phase 2 study showed activity and tolerability of eribulin in advanced or metastatic soft-tissue sarcoma. In this phase 3 study, we aimed to compare overall survival in patients with advanced or metastatic soft-tissue sarcoma who received eribulin with that in patients who received dacarbazine (an active control).
\end{abstract}

Methods We did this randomised, open-label, phase 3 study across 110 study sites in 22 countries. We enrolled patients aged 18 years or older with intermediate-grade or high-grade advanced liposarcoma or leiomyosarcoma who had received at least two previous systemic regimens for advanced disease (including an anthracycline). Using an interactive voice and web response system, an independent statistician randomly assigned (1:1) patients to receive eribulin mesilate $\left(1.4 \mathrm{mg} / \mathrm{m}^{2}\right.$ intravenously on days 1 and 8$)$ or dacarbazine $\left(850 \mathrm{mg} / \mathrm{m}^{2}, 1000 \mathrm{mg} / \mathrm{m}^{2}\right.$, or $1200 \mathrm{mg} / \mathrm{m}^{2}$ [dose dependent on centre and clinician] intravenously on day 1) every 21 days until disease progression. Randomisation was stratified by disease type, geographical region, and number of previous regimens for advanced soft-tissue sarcoma and in blocks of six. Patients and investigators were not masked to treatment assignment. The primary endpoint was overall survival in the intention-to-treat population. The study is registered with ClinicalTrials.gov, number NCT01327885, and is closed to recruitment, but treatment and follow-up continue.

Findings Between March 10, 2011 and May 22, 2013, we randomly assigned patients to eribulin (n=228) or dacarbazine $(n=224)$. Overall survival was significantly improved in patients assigned to eribulin compared with those assigned to dacarbazine (median 13.5 months [95\% CI 10.9-15.6] vs 11.5 months [9.6-13.0]; hazard ratio 0.77 [95\% CI 0.62-0.95]; $p=0.0169)$. Treatment-emergent adverse events occurred in 224 (99\%) of 226 patients who received eribulin and $218(97 \%)$ of 224 who received dacarbazine. Grade 3 or higher adverse events were more common in patients who received eribulin (152 [67\%]) than in those who received dacarbazine (126 [56\%]), as were deaths (10 [4\%] vs $3[1 \%]$ ); one death (in the eribulin group) was considered treatment-related by the investigators.

Interpretation Overall survival was improved in patients assigned to eribulin compared with those assigned to an active control, suggesting that eribulin could be a treatment option for advanced soft-tissue sarcoma.

Funding Eisai.

\section{Introduction}

Sarcomas are rare solid tumours that account for about $1 \%$ of all adult malignancies. About $80 \%$ of sarcomas originate from soft tissue and the remaining $20 \%$ from bone. Together, soft-tissue sarcomas comprise a heterogeneous group of more than 50 different histological subtypes, ${ }^{1-3}$ with an incidence rate of two to five cases per 100000 per year. Survival outcomes in patients with locally advanced, unresectable, or metastatic disease are poor. ${ }^{3-6}$ Treatment options for patients are scarce, both in number and efficacy. First-line treatment is usually chemotherapy with doxorubicin, ifosfamide, or both.

Gemcitabine, an investigative drug in this setting, given alone or in combination with other drugs, has also been studied as a first-line and higher therapy in patients with soft-tissue sarcoma. ${ }^{7-9}$ In a randomised phase 2 study in patients with metastatic soft-tissue sarcoma, the combination of gemcitabine and docetaxel was associated with improved progression-free survival and overall survival compared with gemcitabine alone, but this group had greater toxicity. ${ }^{7}$ Another randomised phase 2 study in previously treated patients with advanced soft-tissue sarcoma showed improved progression-free survival and overall survival with the combination of gemcitabine and dacarbazine than with dacarbazine alone. ${ }^{10}$ Second-line treatment options include ifosfamide, dacarbazine, trabectedin, or pazopanib; other drugs are occasionally used in specific sarcoma subtypes despite no regulatory approval for this indication. ${ }^{2,3,11}$ However, outcomes with these drugs are generally unsatisfactory because they are associated with low response rates, low progression-free and overall survival, and the risk of treatment-limiting toxic effects. ${ }^{3}$ Therefore, more effective and tolerable treatment options are needed for patients with advanced soft-tissue sarcoma.

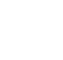

\section{(a)}

atrotine hebruary 10, 2016 http://dx.doi.org/10.1016/ S0140-6736(15)01283-0 See Online/Comment/ http://dx.doi.org/10.1016/ S0140-6736(16)00162-8

Department of General Medical Oncology, University Hospitals Leuven, Leuven Cancer Institute, Leuven, Belgium (Prof P Schöffski MD); Sarcoma Oncology Center, Santa Monica, CA, USA (S Chawla MD); Tisch Cancer Institute, Mount Sinai Medical Center, New York, NY, USA (Prof R G Maki MD); Department of Oncology, Institut Bergonié, Bordeaux, France (A Italiano MD); Department of Medical Oncology, Leiden University Medical Center, Leiden, Netherlands

(Prof H Gelderblom MD); Division of Hematology and Oncology, Massachusetts General Hospital, Boston, MA, USA (E Choy MD); Department of Medical Oncology, Fondazione del Piemonte per I'Oncologia IRCCS, Candiolo, Italy (G Grignani MD); Department of Medical Oncology, Hospital Sírio-Libanês and Instituto do Câncer do Estado de São Paulo, São Paulo, Brazil (V Camargo MD); West German Cancer Center, University of Duisburg-Essen, Essen, Germany (S Baver MD); Yonsei Cancer Center, Yonsei University College of Medicine, Seoul, South Korea

(SY Rha MD); Université Claude Bernard Lyon I, LYRIC INCa-DGOS 4664 \& Centre Léon Bérard, Lyon, France (ProfJ-Y Blay MD); Division of Surgical Oncology \& Thoracic Surgery, Department of Surgery, Mannheim University Medical Center, Mannheim, Germany (P Hohenberger MD); 
Eisai, Woodcliff Lake, NJ, USA (D D'Adamo MD, M Guo PhD); Department of Hematology, UCLA Jonsson Comprehensive Cancer, Los Angeles, CA, USA (B Chmielowski MD); Gustave Roussy, Villejuif, France (A Le Cesne MD); Sarcoma Center, Dana-Farber Cancer Institute and Ludwig Center at Harvard Medical School, Boston, MA, USA (Prof G D Demetri MD); and Department of Sarcoma Medical Oncology, University of Texas, MD Anderson Cancer Center, Houston, TX, USA (Prof SR Patel MD) Correspondence to: Prof Patrick Schöffski, Department of General Medical Oncology, University Hospitals Leuven, Leuven Cancer Institute,

Leuven B-3000, Belgium patrick.schoffski@uzleuven.be

For the protocol see http://www.uzleuven.be/ E7389-G000-309_Final_ protocol_31Jan2011.pdf

\section{Research in context}

\section{Evidence before this study}

In 2010, when this study was designed, we did a PubMed search for reports with the following terms "leiomyosarcoma" OR "liposarcoma" OR "adipocytic sarcoma", and filter for "clinical trial, phase 3 ". Only four published phase 3 studies in patients with leiomyosarcoma or liposarcoma were available (three of the four studies identified investigated combination regimens, while the fourth compared radiotherapy with observation). Only two of the studies included patients with liposarcoma or advanced or metastatic disease. Overall survival was a secondary endpoint in these studies (in trials in which primary and secondary endpoints were clearly defined) and none of the studies reported a significant difference in overall survival between the treatment groups. Median overall survival was not reached in one of the studies. Our more recent PubMed search on Oct 27, 2015, using the above search terms, identified three additional studies, one of these included updated data from the aforementioned study in which median overall survival was not reached. Two of the studies investigated combination regimens, and one compared monotherapy with pazopanib, a multitargeted tyrosine-kinase inhibitor, with placebo in patients with metastatic soft-tissue sarcoma. This study was the most comparable one to the present trial; however, it excluded patients with liposarcoma and its primary endpoint was progression-free survival. Overall survival was a secondary endpoint and no significant difference was noted. None of the three studies had overall survival as a primary endpoint or reported a significant improvement in overall survival.

\section{Added value of this study}

To our knowledge, our study is the first randomised, phase 3 trial of a single-agent systemic therapy with an active control to show a significant improvement in overall survival as the primary endpoint in patients with previously treated advanced leiomyosarcoma and liposarcoma. We noted a statistically significant benefit in overall survival with eribulin compared with the conventional cytotoxic drug dacarbazine, which was deemed to be clinically meaningful by the investigators.

Implications of all the available evidence

The findings from this study suggest that eribulin might be an important treatment option for patients with previously treated liposarcoma or leiomyosarcoma.
Eribulin, a microtubule-dynamics inhibitor, is approved for metastatic breast cancer. Eribulin is a structurally modified analogue of halichondrin B, originally isolated from the marine sponge Halichondria okadai. Its mode of action is distinct from other tubulin inhibitors, and involves binding to specific sites on the growing positive ends of microtubules to inhibit their growth. ${ }^{12,13}$ Eribulin also induces vascular remodelling, suppresses migration and invasion of cancer cells, and reverses the epithelialto-mesenchymal transition in many cancer cell lines..$^{14,15}$ Eribulin showed activity in a non-randomised, phase 2 study of patients with progressive soft-tissue sarcomas who had previously received at most one combination chemotherapy or a maximum of two single drugs for advanced disease..$^{16,17}$ In that study, $12(32 \%)$ of 38 patients with leiomyosarcoma and 15 (47\%) of 32 patients with liposarcoma (also known as adipocytic sarcoma) were progression-free at 12 weeks after the start of therapy, and eribulin was associated with a manageable tolerability profile. ${ }^{17}$

Based on these results, we started this randomised, phase 3 study to assess overall survival with eribulin or an active control (the cytotoxic drug dacarbazine) in pretreated patients with unresectable, advanced, or metastatic leiomyosarcoma or liposarcoma.

\section{Methods}

\section{Study design and participants}

We did this randomised, open-label, multicentre, phase 3 study at 110 study sites in 22 countries across North America, Latin America, Europe, Asia, and Australia (appendix pp 2-10). Eligible patients were aged 18 years or older with histologically confirmed locally recurrent, locally advanced, or metastatic liposarcoma (de-differentiated, myxoid or round-cell, or pleomorphic liposarcoma) or leiomyosarcoma. For eligibility, patients were required to have disease that was not amenable to curative surgery or radiotherapy and that was measurable according to Response Evaluation Criteria in Solid Tumors (RECIST) version $1.1,{ }^{18}$ except for the use of chest radiographs, which could not be used for assessment of chest lesions. Disease progression had to have occurred within 6 months before randomisation after at least two standard systemic regimens for advanced soft-tissue sarcoma, including an anthracycline (unless contraindicated). Previous use of adjuvant and neoadjuvant chemotherapy was allowed but was only considered as a line of treatment if disease progression had been documented within 6 months of its completion. Patients included had an Eastern Cooperative Oncology Group performance status of $0-2$ and adequate organ function. Patients were excluded if they were pregnant, had received any anticancer therapy or major surgery within 21 days before randomisation, or had previously received treatment with eribulin or dacarbazine.

All patients provided written informed consent. The study protocol was approved by independent ethics committees and all relevant institutional review boards for each study site.

\section{Randomisation and masking}

Patients were randomly assigned (1:1) to receive eribulin or dacarbazine by an independent statistician. The randomisation sequence was generated by computer according to the required specifications, and was done 
using an interactive voice and web response system. Randomisation was done in blocks of six and stratified by disease type (liposarcoma or leiomyosarcoma), region (region one, USA and Canada; region two, western Europe, Australia, and Israel; or region three, eastern Europe, Latin America, and Asia), and number of previous regimens for advanced disease (two or more than two). The statistical function lead and study director were masked to treatment assignment until database lock and unblinding. Neither patients nor investigators were masked to treatment assignment.

\section{Procedures}

Eribulin mesilate was given at a dose of $1.4 \mathrm{mg} / \mathrm{m}^{2}$ (equivalent to eribulin $1.23 \mathrm{mg} / \mathrm{m}^{2}$ [expressed as free base]) intravenously over $2-5 \mathrm{~min}$ on day 1 and day 8 of every 21 day cycle. In case of toxic effects, treatment was delayed or the dose reduced to $1.1 \mathrm{mg} / \mathrm{m}^{2}$ or $0.7 \mathrm{mg} / \mathrm{m}^{2}$ (equivalent to eribulin $0.97 \mathrm{mg} / \mathrm{m}^{2}$ or $0.62 \mathrm{mg} / \mathrm{m}^{2}$, respectively [expressed as free base]; appendix $\mathrm{p} 1)$.

Dacarbazine was given at a dose of $850 \mathrm{mg} / \mathrm{m}^{2}$, $1000 \mathrm{mg} / \mathrm{m}^{2}$, or $1200 \mathrm{mg} / \mathrm{m}^{2}$ as an intravenous infusion over 15-60 min on day 1 of every 21 day cycle. The starting dose was selected by the investigator based on each patient's clinical status and institutional preference before randomisation. Haematological toxicities resulted in treatment delays or dose reduction, while clinically relevant hepatic or renal toxicity or hypersensitivity required treatment cessation (appendix $\mathrm{p} 1$ ).

Tumour assessments with CT, MRI, and bone scans were done every 6 weeks from the date of randomisation for the first 12 weeks and every 9 weeks thereafter, or sooner if clinically indicated, until disease progression was confirmed. The follow-up period began immediately after the off-treatment visit, and continued as long as the patient was alive. The survival follow-up was done every 12 weeks, unless the patient withdrew consent or the survival follow-up was terminated by the sponsor. Blood samples for pharmacokinetic assessments were collected on day 1 and day 8 of cycle one and cycle two from all patients receiving eribulin.

\section{Outcomes}

The primary endpoint of this study was overall survival from randomisation until death or last date known alive (censored) in all patients treated with eribulin and dacarbazine. The pre-specified secondary efficacy endpoints were progression-free survival (from randomisation to the date of first documented disease progression or death, whichever occurred first), the number of patients who were alive and progression-free 12 weeks from randomisation, and the number of patients who had clinical benefit (complete response, partial response, durable stable disease $[\geq 11$ weeks $]$ ). Other secondary outcomes were safety and tolerability of eribulin and dacarbazine and characterisation of the population pharmacokinetic profile of eribulin. Safety was assessed by recording and grading all adverse events using the Common Terminology Criteria for Adverse Events version $4 \cdot 02,{ }^{19}$ clinical investigations, and physical examinations. Protocol-specified exploratory endpoints were the proportion of patients with objective response (patients who had a complete or partial response), number of patients with disease control (defined as complete response, partial response, or stable disease), duration of stable disease rate (proportion of patients with stable disease $\geq 11$ weeks), and quality-of-life score between the groups, assessed using the Quality of Life Questionnaire (QLQ) C30 and EQ-5D questionnaires. Other planned exploratory analyses were the relationship between exposure to eribulin and pharmacodynamic biomarkers, efficacy, and adverse events, and identification of blood and tumour biomarkers for safety and efficacy endpoints.

\section{Statistical analysis}

We estimated sample size on the basis of the required number of target events to detect a treatment difference in a comparison of overall survival. The estimated median overall survival in the dacarbazine group was projected to be 6 months on the basis of theoretical historical data. We considered an improvement of 2.5 months to be of clinical importance, which translated

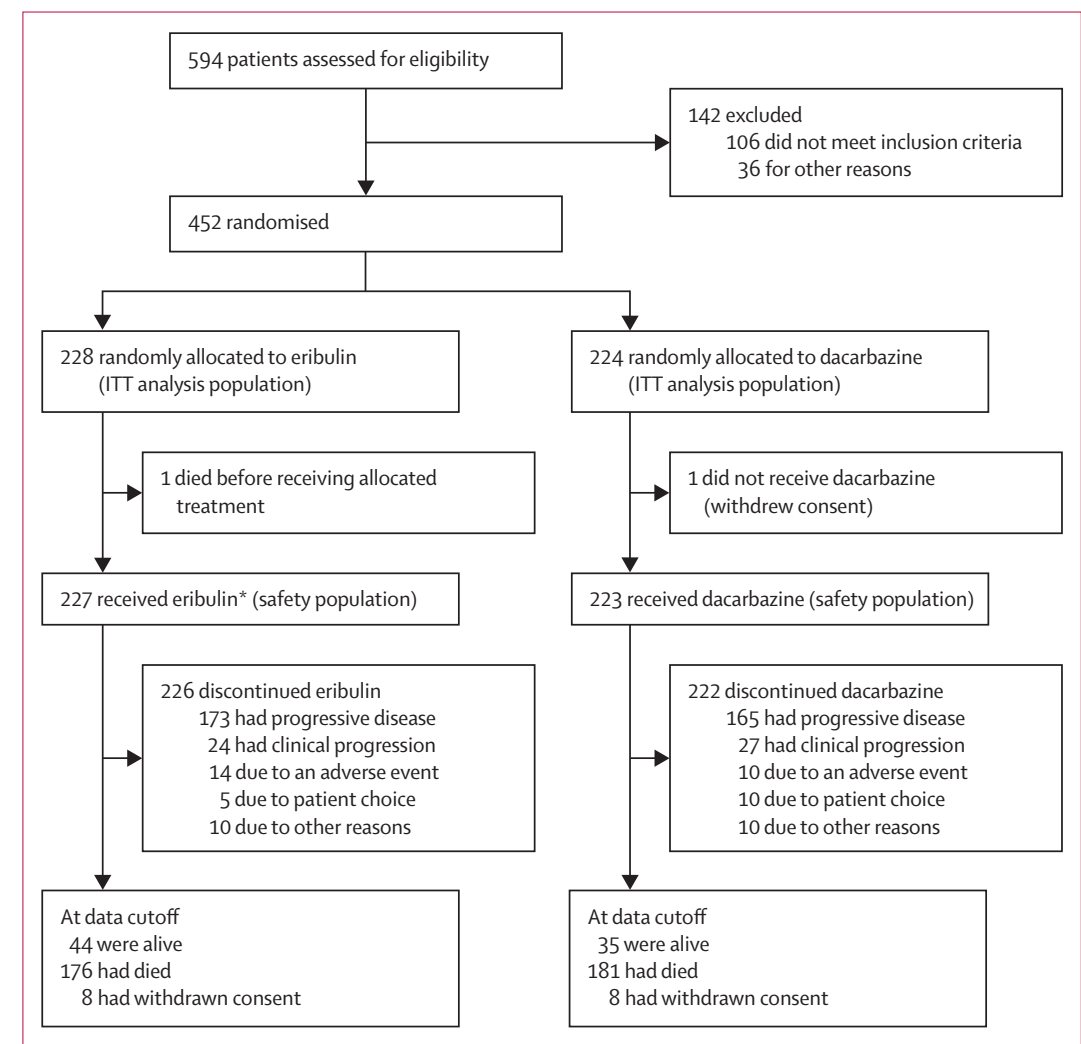

Figure 1: Trial profile

Data cutoff was Jan 2, 2015. ITT=intention-to-treat. *Includes one patient who was assigned to eribulin but treated with dacarbazine. 


\begin{tabular}{|c|c|c|}
\hline & $\begin{array}{l}\text { Eribulin } \\
(\mathrm{n}=228)\end{array}$ & $\begin{array}{l}\text { Dacarbazine* } \\
(n=224)\end{array}$ \\
\hline Median age, years (range) & $56(28-83)$ & $56(24-83)$ \\
\hline$<65$ years & $178(78 \%)$ & $178(79 \%)$ \\
\hline$\geq 65$ years & $50(22 \%)$ & $46(21 \%)$ \\
\hline \multicolumn{3}{|l|}{ Sex } \\
\hline Male & $67(29 \%)$ & $82(37 \%)$ \\
\hline Female & $161(71 \%)$ & $142(63 \%)$ \\
\hline \multicolumn{3}{|l|}{ Race } \\
\hline White & $162(71 \%)$ & $168(75 \%)$ \\
\hline Black or African-American & $6(3 \%)$ & $6(3 \%)$ \\
\hline Asiant & $18(8 \%)$ & $16(7 \%)$ \\
\hline Other or NA & $42(18 \%)$ & $34(15 \%)$ \\
\hline \multicolumn{3}{|l|}{ Ethnic origin } \\
\hline Hispanic or Latino & $23(10 \%)$ & $27(12 \%)$ \\
\hline Not Hispanic or Latino & $170(75 \%)$ & $167(75 \%)$ \\
\hline NA & $35(15 \%)$ & $30(13 \%)$ \\
\hline \multicolumn{3}{|l|}{ ECOG-PS } \\
\hline 0 & $111(49 \%)$ & $90(40 \%)$ \\
\hline 1 & $114(50 \%)$ & $121(54 \%)$ \\
\hline 2 & $3(1 \%)$ & $13(6 \%)$ \\
\hline \multicolumn{3}{|l|}{ Geographical region } \\
\hline Region 1 (USA, Canada) & $87(38 \%)$ & $86(38 \%)$ \\
\hline $\begin{array}{l}\text { Region } 2 \text { (western Europe, } \\
\text { Australia, Israel) }\end{array}$ & $106(46 \%)$ & $105(47 \%)$ \\
\hline $\begin{array}{l}\text { Region } 3 \text { (eastern Europe, Latin } \\
\text { America, Asia) }\end{array}$ & $35(15 \%)$ & $33(15 \%)$ \\
\hline \multicolumn{3}{|l|}{ Disease type } \\
\hline Liposarcoma & $75(33 \%)$ & $78(35 \%)$ \\
\hline Leiomyosarcoma & $152(67 \%)$ & $145(65 \%)$ \\
\hline Other & $1(<1 \%)$ & $1(<1 \%)$ \\
\hline \multicolumn{3}{|l|}{ Liposarcoma histological subtype } \\
\hline De-differentiated & $32(14 \%)$ & $37(17 \%)$ \\
\hline Myxoid or round-cell & $30(13 \%)$ & $26(12 \%)$ \\
\hline Pleomorphic & $13(6 \%)$ & $15(7 \%)$ \\
\hline \multicolumn{3}{|l|}{ Leiomyosarcoma primary site } \\
\hline Uterine & $68(30 \%)$ & $63(28 \%)$ \\
\hline Non-uterine & $83(36 \%)$ & $82(37 \%)$ \\
\hline Unknown & $1(<1 \%)$ & 0 \\
\hline \multicolumn{3}{|l|}{ Tumour grade } \\
\hline High & $150(66 \%)$ & $152(68 \%)$ \\
\hline Intermediate & $77(34 \%)$ & $69(31 \%)$ \\
\hline Not known & $1(<1 \%)$ & $3(1 \%)$ \\
\hline \multicolumn{3}{|l|}{ Type of previous anticancer therapy } \\
\hline Neoadjuvant & $15(7 \%)$ & $10(4 \%)$ \\
\hline Adjuvant & $44(19 \%)$ & $51(23 \%)$ \\
\hline Therapeutic & $224(98 \%)$ & $219(98 \%)$ \\
\hline Maintenance & $8(4 \%)$ & $12(5 \%)$ \\
\hline \multirow[t]{2}{*}{ Unknown } & $3(1 \%)$ & $1(<1 \%)$ \\
\hline & \multicolumn{2}{|c|}{ (Table 1 continues in next column) } \\
\hline
\end{tabular}

to a median overall survival of 8.5 months in patients assigned to eribulin and a hazard ratio $(\mathrm{HR})$ of $0 \cdot 71$. The overall type 1 error was set at 0.05 assuming a two-sided test and power at $90 \%$. On the basis of these assumptions,

\begin{tabular}{|c|c|c|}
\hline & $\begin{array}{l}\text { Eribulin } \\
(n=228)\end{array}$ & $\begin{array}{l}\text { Dacarbazine }{ }^{*} \\
(n=224)\end{array}$ \\
\hline \multicolumn{3}{|c|}{ (Continued from previous column) } \\
\hline \multicolumn{3}{|c|}{ Number of previous chemotherapy regimens $\$$} \\
\hline 0 & 0 & 0 \\
\hline 1 & $2(1 \%)$ & $3(1 \%)$ \\
\hline 2 & $113(50 \%)$ & $103(46 \%)$ \\
\hline$>2$ & $113(50 \%)$ & $118(53 \%)$ \\
\hline \multicolumn{3}{|c|}{ Type of previous chemotherapy received in at least $5 \%$ of patients } \\
\hline Doxorubicin & $179(79 \%)$ & $173(77 \%)$ \\
\hline Gemcitabine & $119(52 \%)$ & $122(54 \%)$ \\
\hline Ifosfamide & $114(50 \%)$ & $112(50 \%)$ \\
\hline Trabectedin & $107(47 \%)$ & $112(50 \%)$ \\
\hline Docetaxel & $97(43 \%)$ & $91(41 \%)$ \\
\hline Pazopanib & $18(8 \%)$ & $17(8 \%)$ \\
\hline Investigational drugs & $18(8 \%)$ & $21(9 \%)$ \\
\hline Epirubicin & $13(6 \%)$ & $14(6 \%)$ \\
\hline Cisplatin & $12(5 \%)$ & $13(6 \%)$ \\
\hline Cyclophosphamide & $12(5 \%)$ & $9(4 \%)$ \\
\hline \multicolumn{3}{|c|}{$\begin{array}{l}\text { Data are n (\%), unless otherwise indicated; due to rounding, percentages might } \\
\text { not total } 100 \text {. NA=not available. ECOG-PS=Eastern Cooperative Oncology Group } \\
\text { performance status. *Dacarbazine was dosed at } 850 \mathrm{mg} / \mathrm{m}^{2}, 1000 \mathrm{mg} / \mathrm{m}^{2} \text {, or } \\
1200 \mathrm{mg} / \mathrm{m}^{2} \text { in this study, based on the investigator's decision before } \\
\text { randomisation for each patient. IIncludes patients from Japan, China, and other } \\
\text { Asian countries. FFor the purposes of eligibility, maintenance therapy was } \\
\text { considered therapeutic. SIncluding neoadjuvant and adjuvant therapy. }\end{array}$} \\
\hline
\end{tabular}

we estimated the event target to be 353 deaths. Assuming an enrolment rate of 20 patients per month, we estimated that we needed about 450 patients to be randomised to observe this number of events.

Safety monitoring was done periodically by the data monitoring committee (DMC) at intervals of no longer than 6 months, starting with the recruitment of the first patient, and as determined by the DMC. An independent statistical reporting team did an interim efficacy analysis when $70 \%$ of events were observed. To maintain an overall type 1 error, we used O'Brien-Fleming stopping boundaries determined by means of the Lan-DeMets approach. With this approach, the relative $\alpha$ spending at the interim and final analyses at 0.7 and 1.0 information fraction were $0 \cdot 0148$ and $0 \cdot 0455$, respectively.

Efficacy was assessed in all randomised patients (intention-to-treat population). All efficacy endpoints other than overall survival were based on the tumour response evaluation according to RECIST 1.1. A two-sided, stratified, log-rank test was used to compare the two groups for the primary endpoint of overall survival and all secondary efficacy endpoints; a nominal significance value of 0.0455 (adjusted for the interim analysis) was used for overall survival analysis. The stratification factors included disease type (liposarcoma or leiomyosarcoma), geography (regions one, two, and three) and number of previous regimens for advanced disease (two or greater than two). Overall survival and 


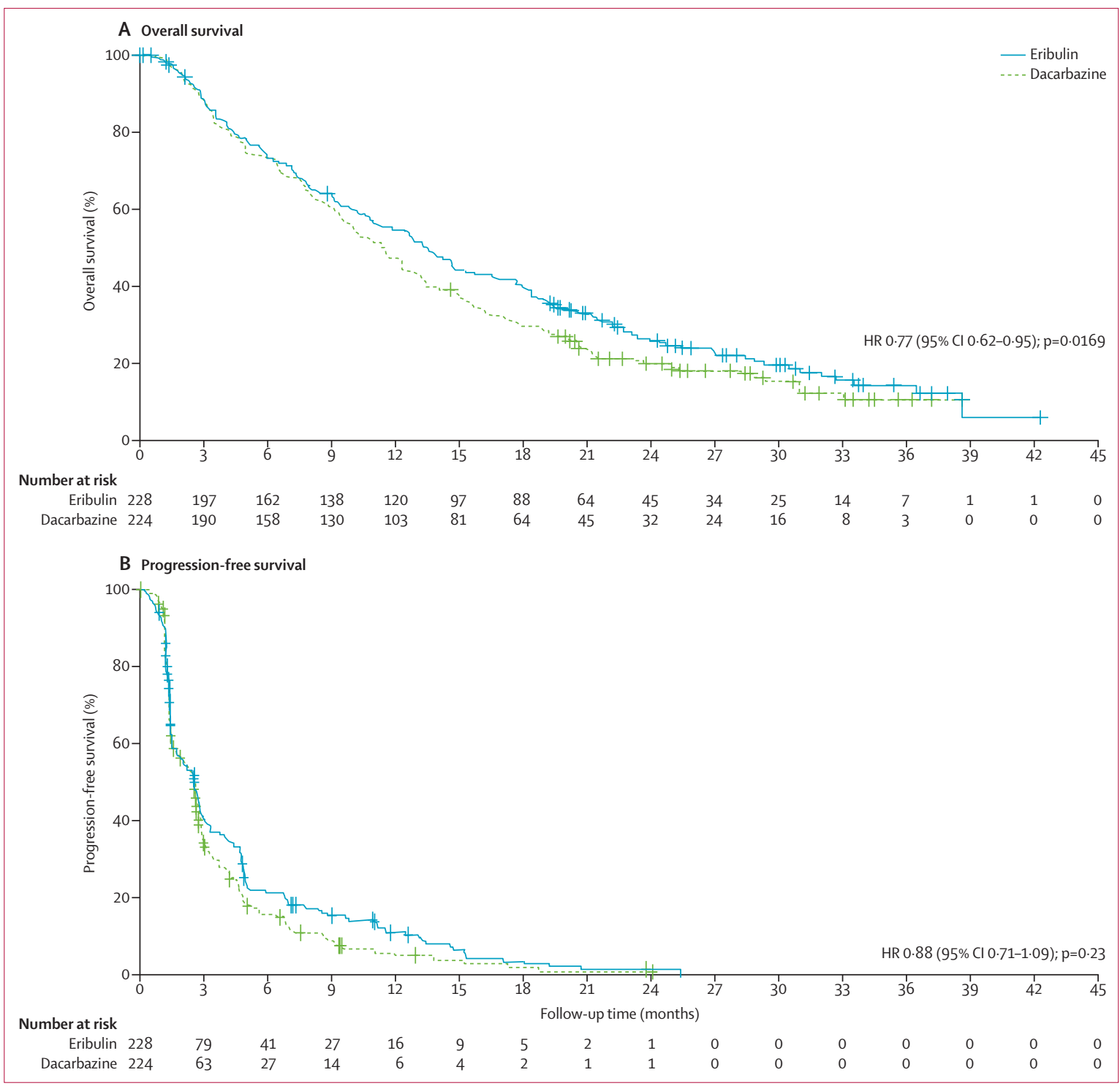

Figure 2: Overall survival (A) and progression-free survival (B) $\mathrm{HR}=$ hazard ratio.

progression-free survival were summarised using KaplanMeier estimates by treatment group and were calculated with two-sided $95 \%$ CIs. For all survival analyses, patients who were lost to follow-up and those who were alive at the date of data cutoff were censored at either the last date known alive or the date of data cutoff, whichever was earliest. Treatment effects were estimated by fitting a Cox proportional hazards model to the overall survival times and stratified as described. An additional exploratory Cox regression model was fitted for planned subgroup analysis in which the HR was stratified as described and, when applicable, with treatment as a covariate. Differences in participants who were progression free at 12 weeks, stable disease for longer than 11 weeks, and had clinical benefit between treatment groups were calculated using the Cochran-Mantel-Haenszel $\chi^{2}$ test with the same strata as the primary endpoint.

The safety analysis set comprised all randomised patients who received at least one dose of the study treatment and had at least one post-baseline safety evaluation. Safety data were summarised separately with descriptive statistics. Treatment-emergent adverse events were described as adverse events that had an onset date of worsening in severity from baseline on or after the first dose of study treatment up to 30 days after the last dose.

All statistical analyses were done after the study was completed; the data cutoff date for analyses was Jan 2, 2015. Statistical analyses were done using SAS version 9.2. This study is registered with ClinicalTrials. gov, number NCT01327885. 


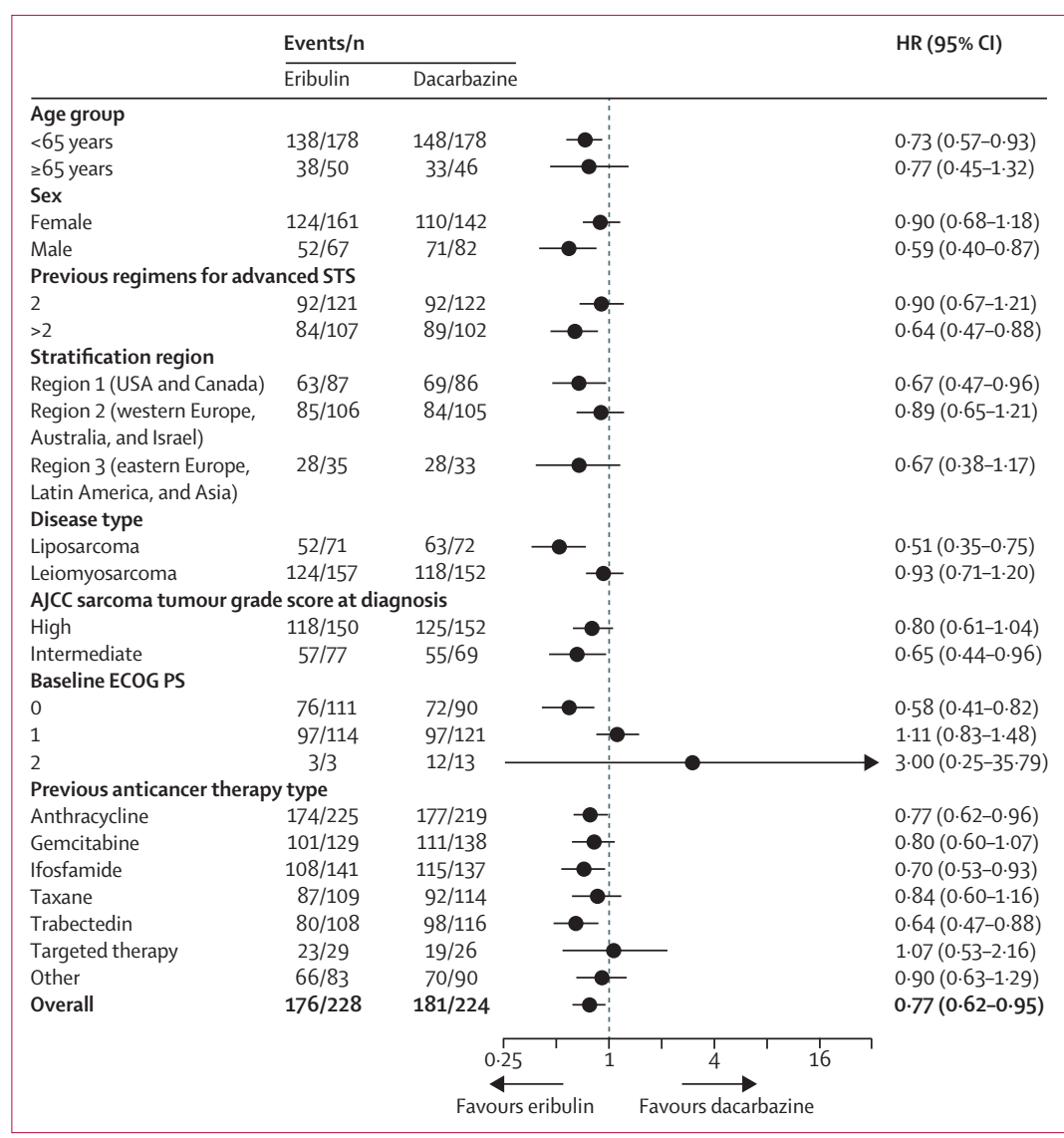

Figure 3: Planned overall survival analyses in various patient subgroups

Overlap with the dashed line at 1 indicates no effect. Median survival shown in appendix p 12. AJCC=American Joint Committee on Cancer. ECOG-PS=Eastern Cooperative Oncology Group performance status. HR=hazard ratio. STS=soft-tissue sarcoma. treatment groups. Patients in the eribulin group had significantly longer overall survival compared with those in the dacarbazine group (HR $0 \cdot 77 ; 95 \%$ CI $0.62-0 \cdot 95$ $\mathrm{p}=0.0169$; figure 2A). Median overall survival was $13 \cdot 5$ months $(95 \%$ CI $10 \cdot 9-15 \cdot 6)$ in the eribulin group and $11 \cdot 5$ months $(9 \cdot 6-13 \cdot 0)$ in the dacarbazine group.

In several pre-specified subgroups, overall survival favoured eribulin (figure 3; appendix p 12). Benefit with eribulin compared with dacarbazine seemed greater in patients with liposarcoma (median overall survival $15 \cdot 6$ [95\% CI $10 \cdot 2-18 \cdot 6]$ vs $8 \cdot 4[5 \cdot 2-10 \cdot 1]$, respectively) than in those with leiomyosarcoma $(12 \cdot 7[9 \cdot 8-14 \cdot 8]$ vs $13 \cdot 0[11 \cdot 3-15 \cdot 1]$; figure 3); however, this study was not powered to draw definitive conclusions from such subgroup analyses.

Median progression-free survival was similar in both treatment groups: 2.6 months $(95 \%$ CI $1 \cdot 9-2 \cdot 8)$ in the eribulin group and 2.6 months $(1 \cdot 8-2.7)$ in the dacarbazine group; and did not significantly differ between the two treatments (HR 0 - 88; 95\% CI 0 -71-1.09; $\mathrm{p}=0 \cdot 23$; figure $2 \mathrm{~B}$ ). Also similar was the proportion of patients who had not progressed at 12 weeks (76 [33\%, $95 \%$ CI 27.2-39.9] patients in the eribulin group vs 64 [29\%, 22 -8-35 - 0] in the dacarbazine group; odds ratio 1.3; 95\% CI $0 \cdot 8-1 \cdot 9 ; \mathrm{p}=0 \cdot 25)$ and the proportion of patients who had clinical benefit $(105$ [46\%, 95\% CI 39.5-52.8] vs 107 [48\%, 95\% CI 41.1-54.5]; odds ratio 0.9; 95\% CI $0 \cdot 7-1 \cdot 4 ; \mathrm{p}=0 \cdot 74)$. No patient had a complete response, and a similar number of patients between groups had partial responses (nine [4\%] in the eribulin group vs 11 [5\%] in the dacarbazine group) or stable disease (119 [52\%] vs 107 [48\%] in the dacarbazine group). The proportion of patients who had an objective response did not differ between treatment groups (nine [4\%] in the eribulin group vs 11 [5\%] in the dacarbazine group; $\mathrm{p}=0 \cdot 62$ ). There were no significant differences between the groups for participants who had disease control (128 [56\%] patients in the eribulin group vs 118 [53\%] in dacarbazine group; $\mathrm{p}=0.438$ ). Duration of stable disease also did not differ. 96 [42\%] patients in eribulin group and $96(43 \%]$ in the dacarbazine group had stable disease for longer than 11 weeks $(p=0 \cdot 90)$. During the treatment period the overall health-related quality of life (Global Health Status scores) did not greatly differ between the eribulin and dacarbazine groups (appendix p 11). The pharmacokinetic profile of eribulin was assessed and will be reported elsewhere. Blood and tumour biomarkers and the relationship between exposure to eribulin and adverse events were assessed and will be reported at a later date.

Treatment-emergent adverse events led to study drug withdrawal of 17 (8\%) of 226 patients in the eribulin safety population and 11 (5\%) of 224 in the dacarbazine safety population; dose reduction in $58(26 \%)$ patients in the eribulin safety population and $32(14 \%)$ in the dacarbazine safety population; and dose interruption in $74(33 \%)$ patients in the eribulin safety population 


\begin{tabular}{|c|c|c|c|c|c|c|}
\hline & \multicolumn{3}{|c|}{ Eribulin $(n=226)$} & \multicolumn{3}{|c|}{ Dacarbazine $(n=224)$} \\
\hline & Grade 1-2 & Grade 3 & Grade 4 & Grade 1-2 & Grade 3 & Grade 4 \\
\hline Any adverse events & $72(32 \%)$ & 88 (39\%) & $54(24 \%)$ & $92(41 \%)$ & $80(36 \%)$ & $43(19 \%)$ \\
\hline Fatigue & $92(41 \%)$ & $7(3 \%)$ & 0 & $83(37 \%)$ & $3(1 \%)$ & 0 \\
\hline Nausea & $89(39 \%)$ & $2(1 \%)$ & 0 & $105(47 \%)$ & $1(<1 \%)$ & 0 \\
\hline Alopecia & $78(35 \%)$ & 0 & 0 & $6(3 \%)$ & 0 & 0 \\
\hline Constipation & $69(31 \%)$ & $2(1 \%)$ & 0 & $57(25 \%)$ & $1(<1 \%)$ & 0 \\
\hline Pyrexia & $61(27 \%)$ & $2(1 \%)$ & 0 & $30(13 \%)$ & $1(<1 \%)$ & 0 \\
\hline Anaemia & $51(23 \%)$ & $14(6 \%)$ & $2(1 \%)$ & $42(19 \%)$ & $22(10 \%)$ & $5(2 \%)$ \\
\hline Asthenia & $43(19 \%)$ & $3(1 \%)$ & $1(1 \%)$ & $44(20 \%)$ & $7(3 \%)$ & 0 \\
\hline Peripheral sensory neuropathy & $42(19 \%)$ & $4(2 \%)$ & 0 & $8(4 \%)$ & 0 & 0 \\
\hline Decreased appetite & $42(19 \%)$ & $2(1 \%)$ & 0 & $41(18 \%)$ & $2(1 \%)$ & 0 \\
\hline Abdominal pain & $41(18 \%)$ & $3(1 \%)$ & $1(<1 \%)$ & $25(11 \%)$ & $8(4 \%)$ & 0 \\
\hline Vomiting & $41(18 \%)$ & $2(1 \%)$ & 0 & $49(22 \%)$ & $1(<1 \%)$ & 0 \\
\hline Headache & $41(18 \%)$ & 0 & 0 & $21(9 \%)$ & 0 & 0 \\
\hline Cough & $39(17 \%)$ & 0 & 0 & $28(13 \%)$ & 0 & 0 \\
\hline Diarrhoea & $37(16 \%)$ & $1(<1 \%)$ & 0 & $34(15 \%)$ & $1(<1 \%)$ & $1(<1 \%)$ \\
\hline Dyspnoea & $31(14 \%)$ & $4(2 \%)$ & $1(<1 \%)$ & $31(14 \%)$ & $3(1 \%)$ & $2(1 \%)$ \\
\hline Back pain & $31(14 \%)$ & $4(2 \%)$ & 0 & $28(13 \%)$ & $3(1 \%)$ & 0 \\
\hline Stomatitis & $29(13 \%)$ & $2(1 \%)$ & 0 & $10(4 \%)$ & $1(<1 \%)$ & 0 \\
\hline Peripheral oedema & $27(12 \%)$ & 0 & 0 & $16(7 \%)$ & $1(<1 \%)$ & 0 \\
\hline Myalgia & $23(10 \%)$ & 0 & 0 & $17(8 \%)$ & 0 & 0 \\
\hline Urinary tract infection & $20(9 \%)$ & $5(2 \%)$ & 0 & $11(5 \%)$ & $1(<1 \%)$ & 0 \\
\hline Neutropenia & $19(8 \%)$ & $45(20 \%)$ & $35(15 \%)$ & $18(8 \%)$ & $20(9 \%)$ & $15(7 \%)$ \\
\hline Hypokalaemia & $17(8 \%)$ & $6(3 \%)$ & 0 & $5(2 \%)$ & $3(1 \%)$ & $1(<1 \%)$ \\
\hline Leukopenia & $13(6 \%)$ & $18(8 \%)$ & $5(2 \%)$ & $13(6 \%)$ & $6(3 \%)$ & $4(2 \%)$ \\
\hline Thrombocytopenia & $12(5 \%)$ & $1(<1 \%)$ & 0 & $28(13 \%)$ & $15(7 \%)$ & $19(8 \%)$ \\
\hline \multicolumn{7}{|c|}{$\begin{array}{l}\text { Maximum grade per patient; shows grade 1-2 events that occurred in at least } 10 \% \text { of patients, and all grade } 3 \text { and } 4 \text { events. Fatal (grade } 5) \text { adverse events in the eribulin } \\
\text { group: respiratory failure }(n=2) \text {, general physical health deterioration }(n=1) \text {, intestinal obstruction }(n=1) \text {, large intestinal perforation }(n=1) \text {, neutropenic sepsis }(n=1) \text {, sept } \\
\text { shock }(n=1) \text {, metastases to lung }(n=1) \text {, acute respiratory failure }(n=1) \text {, and pneumonia aspiration }(n=1) \text {; in the dacarbazine group: respiratory failure }(n=1) \text {, general physic } \\
\text { health deterioration }(n=1) \text {, and cardiac arrest }(n=1) \text {. }\end{array}$} \\
\hline
\end{tabular}

and $72(32 \%)$ in the dacarbazine safety population. 224 (99\%) of 226 patients given eribulin and 218 (97\%) of 224 given dacarbazine had treatment-emergent adverse events (table 2); most of which were treatment-related (210 [93\%] given eribulin and 203 [91\%] given dacarbazine). The incidence of grade 3 or greater events was higher in the eribulin safety population (152 [67\%]) compared with the dacarbazine safety population (126 [56\%]). Neutropenia and leukopenia were more common in patients given eribulin, whereas anaemia and thrombocytopenia were more common in those given dacarbazine (table 2). The incidence of grade 3 or higher febrile neutropenia was low in both populations.

13 patients died from treatment-emergent adverse events (table 2). Of ten events in the eribulin safety population, only one (neutropenic sepsis) was considered by the investigators to be possibly related to therapy, and another event (septic shock) was considered to be possibly treatment-related by the sponsor (but not by the investigators). None of the three deaths in the dacarbazine safety population were considered by the investigators to be related to treatment.

In a post-hoc analysis we found that the administration of post-study chemotherapies was similar between the treatment groups (158 [69\%] of 228 patients in the eribulin group, 141 [63\%] of 224 in the dacarbazine group) except for the expected higher number of patients in the eribulin group (78 [34\%]) who received dacarbazine outside of this trial (appendix $p 1$ ). The post-study use of radiotherapy and surgery was similar between the treatment groups (appendix p 1). Post-hoc analyses were done to assess overall survival by planned dacarbazine dose, and overall survival outcomes were similar irrespective of the planned dacarbazine dose (appendix p 11).

\section{Discussion}

This randomised, phase 3 study of a systemic therapy led to a significant improvement in its primary endpoint, overall survival, in patients with previously treated advanced leiomyosarcoma or liposarcoma. The observed 2 month difference in overall survival between the 
treatment groups to favour eribulin was statistically significant and deemed to be clinically meaningful by the investigators.

Dacarbazine was chosen as the active comparator (control) in this study because it represents an acceptable and active treatment option in this setting after failure of other standard therapies, is widely available, and is commonly used, as shown by the high proportion of patients in this study who received dacarbazine after the study (appendix p 1). On the basis of clinical evidence from its use as monotherapy or in combination with other drugs, dacarbazine is recommended by clinical practice guidelines as a treatment option for advanced soft-tissue sarcoma. ${ }^{2,11}$ Investigators of a phase 3 study for trabectedin in patients with advanced soft-tissue sarcoma also used dacarbazine as the control drug. ${ }^{20}$ In the present study, we allowed three different dosages for dacarbazine to accommodate various institutional guidelines and investigator preferences. Our post-hoc analysis indicates that the different planned dacarbazine doses were associated with similar overall survival outcomes (appendix p 11). On the basis of similar historical data and in consultation with sarcoma experts, an overall survival of 6 months was anticipated in patients treated with dacarbazine in this study, at the time of its design in 2010. However, as in other recent studies, ${ }^{21,22}$ overall survival in both study groups surpassed initial predictions (median overall survival 11.5 months with dacarbazine vs 13.5 months with eribulin), indicating that our original prediction might have been an underestimate.

Pre-planned subgroup analyses suggested that the overall survival benefit with eribulin was independent of age, sex, number of prior regimens for advanced disease, and geographic region. The overall survival outcomes seemed unaffected by the type of previous anticancer therapy. In subgroup analyses, benefit with eribulin was particularly strong in patients with liposarcoma compared with those with leiomyosarcoma; however, the liposarcoma subgroup was small and the study was not statistically powered for comparisons between the subgroups.

The benefit in overall survival with eribulin in this study was observed despite a lack of statistically significant differences between the treatment groups in secondary endpoints of progression-free survival, participants whose tumours had not progressed at 12 weeks, and participants with clinical benefit. A greater effect of eribulin on overall survival compared with progression-free survival has been reported previously in studies in patients with metastatic breast cancer. ${ }^{23,24}$ These data indicate that the secondary endpoints in this study could underestimate the efficacy of eribulin, and therefore might not be an accurate predictor of overall survival benefit in patients with leiomyosarcoma and liposarcoma. The proportion of patients who received post-study therapy was similar between the two treatment arms in this study, except for the higher proportion of patients in the eribulin group who went on to receive dacarbazine after the study, as expected. However, this difference is not judged sufficient to account for the observed benefit in overall survival with eribulin treatment. The positive effect on overall survival compared with absence of effect on progression-free survival might be attributed to the biological effects of eribulin on tumour vascularisation, microenvironment, and metastases..$^{14,15}$ The tissue samples collected from most patients enrolled in this study have not yet been analysed, and further investigation of these could help to show the relevance of these effects.

Treatment-emergent adverse events were consistent with the known safety profiles for eribulin and dacarbazine. Eribulin was associated with a manageable toxicity profile, similar to previous findings. ${ }^{17}$ No new or unexpected safety signals were noted. Most patients had maintained or improved quality of life, with no important differences between the two treatment groups.

Our study is distinct from other recently published phase 3 studies in patients with soft-tissue sarcoma that mostly assessed combination regimens, ${ }^{25-27}$ and rarely used overall survival as the primary endpoint. . $2,25,27^{2}$ Although results from a phase 3 study comparing pazopanib with placebo (PALETTE; NCT00753688) were published in 2012, its study population excluded patients with liposarcoma. ${ }^{22}$ The PALETTE study also did not report a significant increase in overall survival-a secondary endpoint-with pazopanib compared with placebo. ${ }^{22}$ On the basis of the strong evidence for its use presented in this study, eribulin merits further exploration in less common subtypes of soft-tissue sarcoma and in earlier lines of treatment, either alone or in combination with other drugs, in future studies.

The absence of masking is a potential limitation of this study. Both quality of life and objective response rate were exploratory endpoints in this study. The exploratory nature of these endpoints might also be considered to be potential limitations of this study because these can influence treatment decisions. However, overall survival is considered the gold-standard endpoint in oncology studies, and a 2 month survival advantage conferred by eribulin was statistically significant and deemed to be clinically relevant.

In conclusion, we show that eribulin improved overall survival in patients with advanced soft-tissue sarcoma compared with an active control, dacarbazine. Our findings suggest that eribulin might be an important treatment option for patients with previously treated liposarcoma or leiomyosarcoma.

\section{Contributions}

PS put forward the concept for this study to Eisai, and contributed to the design of the study, data collection, data interpretation, and writing of the manuscript. All authors contributed to data analysis, data interpretation, and writing of the manuscript. All authors provided final approval of the manuscript for submission. 


\section{Declaration of interests}

ALC declares personal fees from Novartis, Pharmamar, Pfizer, and GlaxoSmithKline. GD declares grants from Eisai, Pfizer, Threshold Pharmaceuticals, Janssen Oncology, Bayer, Novartis, and GlaxoSmithKline; personal fees from Pfizer, Lilly, EMD Serono, Threshold Pharmaceuticals, Sanofi Oncology, GlaxoSmithKline, Saiichi-Sankyo, Ariad, AstraZeneca, Ziopharm, Polaris Pharmaceuticals, Kolltan Pharmaceuticals, Blueprint Medicines, Caris Life Sciences, Champions Oncology, and Bessor Pharmaceuticals; and has the following declarations: Kolltan Pharmaceuticals (stock options), Blueprint Medicines (stock, stock options, and service on the Board of Directors), Caris Life Sciences (stock options), Champions Oncology (stock options), and Bessor Pharmaceuticals (stock options). SRP is a consultant for Novartis, EMD Serono, Johnson and Johnson, and CytRx; and is the data safety and monitoring committee chair for CytRx. All other authors declare no competing interests. All institutions were compensated for participation in this study.

\section{Acknowledgments}

We thank the patients, their families, the investigators, and the teams who participated in this trial; and Shilpa Aggarwal and Brian Falcone of Oxford PharmaGenesis for providing editorial assistance (funded by Eisai). A complete list of all recruiting sites and the principal investigators is provided in the appendix. Presented in part at the American Society of Clinical Oncology Annual Meeting; May 29-June 2, 2015; Chicago, IL, USA.

\section{References}

1 Fletcher CD, Bridge JA, Hogendoorn P, Mertens F. WHO classification of tumours of soft tissue and bone. 4 th edn. World Health Organization, 2013.

2 National Comprehensive Cancer Network clinical practice guidelines in oncology (NCCN guidelines). Soft tissue sarcoma. Version 1. Fort Washington, PA: National Comprehensive Cancer Network, 2015.

3 Schöffski P, Cornillie J, Wozniak A, Li H, Hompes D. Soft tissue sarcoma: an update on systemic treatment options for patients with advanced disease. Oncol Res Treat 2014; 37: 355-62.

4 Mastrangelo G, Coindre JM, Ducimetière F, et al. Incidence of soft tissue sarcoma and beyond: a population-based prospective study in 3 European regions. Cancer 2012; 118: 5339-48.

5 Stiller CA, Trama A, Serraino D, et al. Descriptive epidemiology of sarcomas in Europe: report from the RARECARE project. Eur J Cancer 2013; 49: 684-95.

6 Nedea EA, DeLaney TF. Sarcoma and skin radiation oncology. Hematol Oncol Clin North Am 2006; 20: 401-29.

7 Maki RG, Wathen JK, Patel SR, et al. Randomized phase II study of gemcitabine and docetaxel compared with gemcitabine alone in patients with metastatic soft tissue sarcomas: results of sarcoma alliance for research through collaboration study 002. J Clin Oncol 2007; 25: 2755-63.

8 Munhoz RR, D'Angelo SP, Gounder MM, et al. A Phase Ib/II study of gemcitabine and docetaxel in combination with pazopanib for the neoadjuvant treatment of soft tissue sarcomas. Oncologist 2015; 20: $1245-46$.

9 Dickson MA, D'Adamo DR, Keohan ML, et al. Phase II trial of gemcitabine and docetaxel with bevacizumab in soft tissue sarcoma. Sarcoma 2015; 2015: 532478 .

10 García-Del-Muro X, López-Pousa A, Maurel J, et al. Randomized phase II study comparing gemcitabine plus dacarbazine versus dacarbazine alone in patients with previously treated soft tissue sarcoma: a Spanish Group for Research on Sarcomas study. J Clin Oncol 2011; 29: 2528-33.

11 ESMO/European Sarcoma Network Working Group. Soft tissue and visceral sarcomas: ESMO clinical practice guidelines for diagnosis, treatment and follow-up. Ann Oncol 2014; 25 (suppl 3): iii102-12.
12 Dybdal-Hargreaves NF, Risinger AL, Mooberry SL. Eribulin mesylate: mechanism of action of a unique microtubule-targeting agent. Clin Cancer Res 2015; 21: 2445-52.

13 Smith JA, Wilson L, Azarenko O, et al. Eribulin binds at microtubule ends to a single site on tubulin to suppress dynamic instability. Biochemistry 2010; 49: 1331-37.

14 Funahashi Y, Okamoto K, Adachi Y, et al. Eribulin mesylate reduces tumor microenvironment abnormality by vascular remodeling in preclinical human breast cancer models. Cancer Sci 2014; 105: 1334-42.

15 Yoshida T, Ozawa Y, Kimura T, et al. Eribulin mesilate suppresses experimental metastasis of breast cancer cells by reversing phenotype from epithelial-mesenchymal transition (EMT) to mesenchymal-epithelial transition (MET) states. Br J Cancer 2014; 110: $1497-505$.

16 E7389 administered as an IV bolus infusion day 1 and day 8 every 3 weeks in pre-treated patients with advanced and/or metastatic soft tissue sarcoma (NCT00413192). ClinicalTrials.gov. https:// clinicaltrials.gov/ct2/show/NCT00413192 (accessed July 22, 2015).

17 Schöffski P, Ray-Coquard IL, Cioffi A, et al. Activity of eribulin mesylate in patients with soft-tissue sarcoma: a phase 2 study in four independent histological subtypes. Lancet Oncol 2011; 12: 1045-52.

18 Eisenhauer EA, Therasse P, Bogaerts J, et al. New response evaluation criteria in solid tumours: revised RECIST guideline (version 1.1). Eur J Cancer 2009; 45: 228-47.

19 Common Terminology Criteria for Adverse Events (CTCAE). Version 4.0. U.S. Department of Health and Human Services, National Institutes of Health, and National Cancer Institute, 2009. https://www.acrin.org/portals/0/administration/regulatory/ CTCAE_4.02_2009-09-15_quickreference_5x7.pdf (accessed July 22, 2015).

20 Demetri GD, von Mehren M, Jones RL, et al. Efficacy and safety of trabectedin or dacarbazine for metastatic liposarcoma or leiomyosarcoma after failure of conventional chemotherapy: results of a phase III randomized multicenter clinical trial. J Clin Onco 2015; Sep 14. DOI: 10.1200/JCO.2015.62.4734

21 Paz-Ares L, López-Pousa A, Poveda A, et al. Trabectedin in pre-treated patients with advanced or metastatic soft tissue sarcoma: a phase II study evaluating co-treatment with dexamethasone. Invest New Drugs 2012; 30: 729-40.

22 van der Graaf WT, Blay JY, Chawla SP, et al. Pazopanib for metastatic soft-tissue sarcoma (PALETTE): a randomised, double-blind, placebo-controlled phase 3 trial. Lancet 2012; 379: 1879-86.

23 Cortes J, O'Shaughnessy J, Loesch D, et al. Eribulin monotherapy versus treatment of physician's choice in patients with metastatic breast cancer (EMBRACE): a phase 3 open-label randomised study. Lancet 2011; 377: 914-23.

24 Kaufman PA, Awada A, Twelves C, et al. Phase III open-label randomized study of eribulin mesylate versus capecitabine in patients with locally advanced or metastatic breast cancer previously treated with an anthracycline and a taxane. J Clin Oncol 2015; 33: 594-601.

25 Blay JY, Papai Z, Tolcher AW, et al. Ombrabulin plus cisplatin versus placebo plus cisplatin in patients with advanced soft-tissue sarcomas after failure of anthracycline and ifosfamide chemotherapy: a randomised, double-blind, placebo-controlled, phase 3 trial. Lancet Oncol 2015; 16: 531-40.

26 Judson I, Verweij J, Gelderblom H, et al. Doxorubicin alone versus intensified doxorubicin plus ifosfamide for first-line treatment of advanced or metastatic soft-tissue sarcoma: a randomised controlled phase 3 trial. Lancet Oncol 2014; 15: 415-23.

27 Issels RD, Lindner LH, Verweij J, et al. Neo-adjuvant chemotherapy alone or with regional hyperthermia for localised high-risk soft-tissue sarcoma: a randomised phase 3 multicentre study. Lancet Oncol 2010; 11: 561-70. 